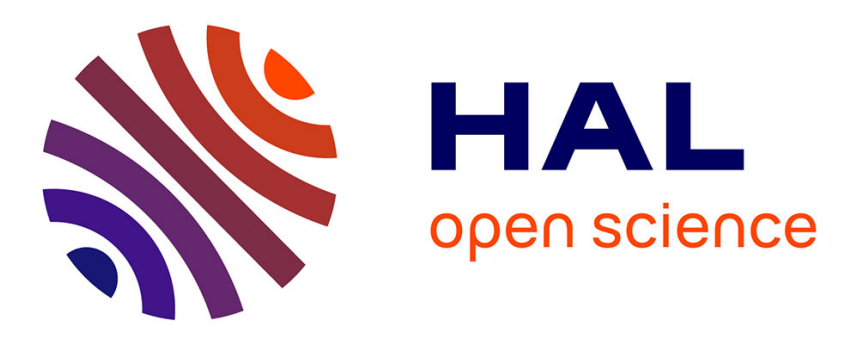

\title{
A Micro-Embolic Energy Detector based on Sub-band Decomposition
}

Maroun Geryes, Sébastien Ménigot, Walid Hassan, Ali Mcheick, Marilys Almar, Benoit Guibert, Corinne Gautier, Jamal Charara, Jean Marc Girault

\section{- To cite this version:}

Maroun Geryes, Sébastien Ménigot, Walid Hassan, Ali Mcheick, Marilys Almar, et al.. A MicroEmbolic Energy Detector based on Sub-band Decomposition. MECBME, Oct 2016, Beirut, Lebanon. pp.54-58, 10.1109/MECBME.2016.7745407 . hal-01378982

\section{HAL Id: hal-01378982 \\ https://hal.science/hal-01378982}

Submitted on 24 Nov 2016

HAL is a multi-disciplinary open access archive for the deposit and dissemination of scientific research documents, whether they are published or not. The documents may come from teaching and research institutions in France or abroad, or from public or private research centers.
L'archive ouverte pluridisciplinaire $\mathbf{H A L}$, est destinée au dépôt et à la diffusion de documents scientifiques de niveau recherche, publiés ou non, émanant des établissements d'enseignement et de recherche français ou étrangers, des laboratoires publics ou privés. 


\title{
A Micro-Embolic Energy Detector based on Sub-band Decomposition
}

\author{
Maroun Geryes* ${ }^{\dagger}$, Sébastien Ménigot*, Walid Hassan ${ }^{\ddagger}$, Ali Mcheick ${ }^{\dagger}$ Marilys Almar $^{\S}$ \\ Benoit Guibert ${ }^{\S}$ Corinne Gautier ${ }^{\Uparrow}$ Jamal Charara ${ }^{\dagger}$ and Jean-Marc Girault * \\ * Université François Rabelais de Tours, UMR "Imagerie \& Cerveau" Inserm U930, Tours, France \\ †Department of Physics and Electronics \\ Faculty of Sciences I, Lebanese University \\ $\ddagger$ Thomson Reuters

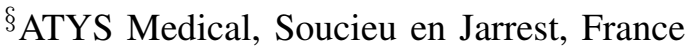 \\ ${ }^{\top} \mathrm{CHU}$ de Lille, Lille, France
}

\begin{abstract}
Cerebrovascular Accidents can be caused by cerebral emboli blocking brain blood vessels. Analysis of transcranial Doppler signals practically aids the detection of emboli. Signal processing methods have been proposed for emboli detection. In this study, we introduce a new micro-embolic energy detector composed of $\mathrm{N}$ detectors associated to $\mathrm{N}$ Doppler frequency sub-bands. To test our detectors, we propose a training phase during which we adjust the optimal number of sub-bands and detection thresholds and a testing phase through which we measure performances. Using real signals, we show that in terms of the number of sub-bands, 4 sub-bands provide the highest detection rate and lowest false alarm. Moreover, compared to standard detectors, the 4 sub-band energy detector reduces the false alarm rate from $44 \%$ to $36 \%$, increases the detection rate from $66 \%$ to $79 \%$ and augments the Embolus to Blood Ratio from $24 \mathrm{~dB}$ to $40 \mathrm{~dB}$. This new energy detector permits detecting smallest micro-emboli, precursors of coming large emboli with high stroke risks.

Keywords: Cerebrovascular Accidents; transcranial Doppler; micro-emboli detection; sub-band decomposition ${ }^{1}$
\end{abstract}

\section{INTRODUCTION}

Strokes occur when blood flow to a part of the brain is suddenly stopped either by a rupture or a blockage of a blood vessel. Strokes are the second cause of mortality worldwide after cancer [1]. Therefore, strokes represent a major death threat and are a public health issue for which a number of research events are initiated in order to find solutions to reduce its occurrence rate and boost stroke-survival chances.

About one third of strokes have embolic nature [2]. Emboli in cerebral circulation can occlude cerebral arteries leading to strokes. This is known as cerebral embolism.

The Transcranial Doppler system is widely considered an effective solution for stroke diagnosis [3]. The TCD measures the velocity of blood flow in the brain's blood vessels and also the relative changes in the flow. Transcranial Doppler diagnoses are based on the detection of altered blood flow velocity, absence of blood flow, or changes in the spectral waveform. The TCD is the only non-invasive examination that provides reliable evaluation of cerebral embolism. TCD allows detection of embolic events from the Doppler signal in the form of high intensity transient signals (HITS) superimposed on the Doppler signal backscattered by the blood.

Detection of small micro-emboli, precursors of coming large emboli, is central to the early anticipation and prevention of strokes. Visualization of the spectrogram on the TCD system accompanied with audible detection is considered as the gold standard of micro-emboli detection. From a signal processing standpoint, almost all commercial TCD systems are based on Short Time Fourier Transform calculations. Standard methods, where detection is performed over the whole Doppler frequency spectrum, are mainly suitable for the detection of intense embolic signals. Their performance in the detection of low intensity micro-embolic signals is mainly poor.

In this paper, we propose a new energy detection approach based on sub-band decomposition. The new energy detector we present is composed of $\mathrm{N}$ detectors associated to $\mathrm{N}$ Doppler frequency sub-bands. The underlying idea is that by using an energy detector in each sub-band of the Doppler spectrum, we reduce the number of Red Blood Cells contributing in the background Doppler energy. Thus we amplify the contrast between the micro-embolus Doppler energy and the background Doppler energy. This detector should improve the detectability of the smallest micro-emboli and advance the accurateness of

${ }^{1}$ DOI : 10.1109/MECBME.2016.7745407

(C) 2016 IEEE. Reprinted, with permission, from Maroun Geryes, Sbastien Mnigot, Walid Hassan, Ali Mcheick, Marilys Almar, Benoit Guibert, Corinne Gautier, Jamal Charara and Jean-Marc Girault, A Micro-Embolic Energy Detector based on Sub-band Decomposition, 2016 3rd Middle East Conference on Biomedical Engineering (MECBME), 2016. This material is posted here with permission of the IEEE. Such permission of the IEEE does not in any way imply IEEE endorsement of any of the Universit Franois Rabelais de Tours' products or services. Internal or personal use of this material is permitted. However, permission to reprint/republish this material for advertising or promotional purposes or for creating new collective works for resale or redistribution must be obtained from the IEEE by writing to pubs-permissions@ieee.org. 


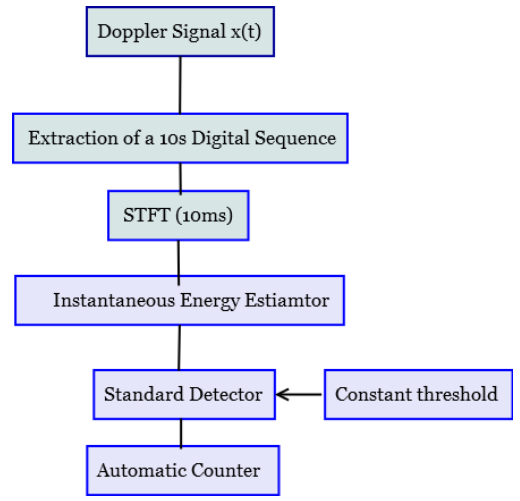

Figure 1: Standard embolus detection system

detection. Micro-emboli detection using sub-band decomposition was introduced by using wavelet decomposition as in [4], [5], [6], and [7]. Unlike previously introduced wavelet-based detections, we implement sub-band decomposition performed after application of a bank of $\mathrm{n}$ ideal frequency filters.

\section{Sub-band Energy Detection of Micro-emboli}

\section{A. Instantaneous Energy Detection of Micro-Embolic Signals}

The presence of micro-emboli can be reliably detected through instantaneous energy calculation. Energy of the backscattered Doppler signal is proportional to the size of the scatterer to power of 6 [5]. In other words, the energy of a micro-embolus, which is larger than a Red Blood Cell, is higher in the Doppler energy signal. In fact, the energy returned by a micro-embolus is greater than that returned by billions of red blood cells, whatever the circulation speed is.

Actually, most automatic detectors are majorly based on energy criteria. Moreover, these detectors, employed by commercial TCD systems, are based on the Short Time Fourier Transform, an adapted form of the Fourier transform that analyzes only a small segment of the signal at a time -a technique called windowing of the signal. The STFT provides a time-frequency representation known as the spectrogram, from which visual micro-embolic detection is performed. The instantaneous energy can be assessed from STFT-based frequency estimators. These estimators are short-term estimators and the calculation is carried out using a sliding window $w(t)$. They are formally written as:

$$
S(t, f)=\left|\int x(\tau) w^{*}(t-\tau) \exp ^{-j w t} d \tau\right|^{2}
$$

where $x(t)$ is the analyzed Doppler signal, $w(t)$ is a sliding window and $*$ stands for complex conjugation. The instantaneous energy at a fixed time $t$ can be obtained from STFT frequency estimators in (1) by:

$$
E(t)=\int S(t, f) d f
$$

In our implementations, STFT and instantaneous energy calculations are carried out repetitively on 10 second segments extracted from the Doppler signal. After a preliminary experimental phase and based on our previous experiences, the STFT is performed using a 64 point Hamming window with an overlap of $65 \%$.

Energy detection of micro-embolic signals is based on the following binary test:

$$
E(t)\left\{\begin{array}{l}
>\lambda, \text { Hypothesis } H_{1} \\
<\lambda, \text { Hypothesis } H_{0}
\end{array}\right.
$$

If the instantaneous energy $\mathrm{E}(\mathrm{t})$ is greater than the detection threshold then an embolus is detected (hypothesis $H_{1}$ ). In this case the automatic energy detector is triggered. Otherwise the automatic detector remains mute and no embolus is detected (hypothesis $H_{0}$ ).

\section{B. Standard Detection}

In standard energy detectors, the detection process in (3) is performed over the whole Doppler spectral band. The instantaneous energy calculated over the whole spectrum is compared to the threshold. This simple detection process is demonstrated in figure 1. The threshold is a constant threshold. This constant threshold is empirical; i.e. it is fixed empirically by the user for the entire examination. It is patient-, operator-, and device-dependent. It is usually set above the maximal detected energy of the Doppler signal when no embolus is present, where this maximum energy always happens during the heart systolic phase. Generally, for automatic detection, the constant threshold is set between 3 to $9 d B$ above the highest energy and depends on the TCD system calibration. 


\section{Detection by Sub-band Decomposition}

Factually, the micro-embolus travels on a single line in the artery. Therefore, the Doppler micro-embolic signal can be considered as 'narrow band' signal corresponding to the velocity of the passing micro-emboli, compared to the 'wide band' Doppler blood flow signal which includes the velocities of all the red blood cells traveling along the various lines in the artery [5]. The energy in the latter 'narrow band' reflects the energy backscattered by the traveling micro-embolus as well as the energy backscattered by all Red Blood Cells (RBC) traveling on the same velocity line as the micro-embolus.

Indeed, if we propose to reduce the frequency band, through a sub-band decomposition process, we can reduce the number of RBC contributing in the background Doppler signal. Only RBC traveling at the same speed as the micro-embolus will be considered instead of considering all RBCs present in the region of interest. Thus sub-band decomposition, provides a suitable tool to enhance the contrast between the micro-embolic energy signature and the background Doppler signal. Subband decomposition allows to decompose the whole Doppler spectral band into several channels. Through this decomposition, we reduce the bandwidth in which detection is applied. This in turn allows to reduce the background (RBC) energy in the considered bandwidth.

In the sub-band decomposition process, we compute the energy of the Doppler signal $y(t)$ decomposed in $\mathrm{N}$ sub-bands. The energy is assessed in each channel $\mathrm{n}$ where $E_{n}$ will denote the instantaneous energy calculated in the $n^{t h}$ sub-band. The threshold is also assessed in each sub-band $\mathrm{n}$ and is denoted by $\lambda_{n}$.

After sub-band decomposition, the Doppler signal $y(t)$ can be expressed as follows:

$$
y(t)=\sum_{n=1}^{N_{s b}} y_{n}(t)
$$

where $N_{s b}$ is the total number of channels (sub-bands) and $y_{n}(t)$ is the signal in the $n^{\text {th }}$ sub-band.

Also, the total instantaneous energy over the whole spectral band can be obtained by summing the contribution of all channels:

$$
E(t)=\sum_{n=1}^{N_{s b}} E_{n}(t)
$$

Sub-band detection procedure is demonstrated in the block diagram in figure 2. Simply speaking, the same procedure as standard detection is performed in each sub-band.

\section{ACQUISITION OF THE DOPPLER SIGNAL}

Transcranial Doppler is considered to be the most suitable system for the detection of micro-emboli in the cerebral circulation. TCD is a harmless and safe technique. It is non-invasive and non-ionizing. While most detection methods are initially designed to function on standard TCD systems, in our study we operate a new TCD generation known as the transcranial Holter. This Holter system, also known as TCD-X, has been implemented by a French company called Atys Medical (Soucieu en Jarrest, France). Unlike standard TCD systems, the Holter version allows up to 5 hours of patient monitoring and thus offers more validity for micro-embolus detection. Using the Holter system, the patient is no longer attached to a TCD and does not need to be laying on a bed. Patient monitoring can be done under naturalistic conditions with the possibility of patient motion and action.

The Holter recordings were carried out at the Centre Hospitalier Régional Universitaire (CHRU) de Lille hospital in Lille, France. Patients with carotid stenosis were chosen for the study. Informed agreement for Holter monitoring was obtained from all patients. The Holter probe emits an Ultrasound wave with a frequency of $1.5 \mathrm{MHz}$ to the middle cerebral artery of the patients. The pulse repetition frequency (PRF) is $6.4 \mathrm{kHz}$ and the Ultrasound power is $50 \mathrm{~mW} / \mathrm{cm}^{-2}$.

Primarily, the Holter recordings are converted into audio-wave files. The gold standard of detection is then constituted. Audible detection is performed at the normal speed and at half the normal speed. Micro-emboli are detected based on their characteristic click or chirping sound. Simultaneously, visualization of the spectrogram is performed. The positions in time of micro-embolic events audibly and visually detected are noted.

Our 17 acquired Doppler signals are divided into two groups. The first group of signals (8 signals) are dedicated to achieve the training phase. The training phase, a preliminary significant step, allows adjusting the best parameters and standards that mainly maximize the detection rate and minimize the false alarm rate. The second group of signals ( 9 signals) are dedicated for testing the different detectors and obtaining the associated results.

To evaluate the results of the detectors, we compare these results to the gold standard of detection (audible and visual detection) established initially.

The basic statistical tools considered for our evaluation are:

1) Detection Rate

2) False Alarm Rate (FAR)= Number of False Alarm (NFA) / total Number of Detections (ND)

NFA is the instance of detecting an embolus where in reality no embolus crossed and can be referred to as false positive detection. ND is the total number of detections. 


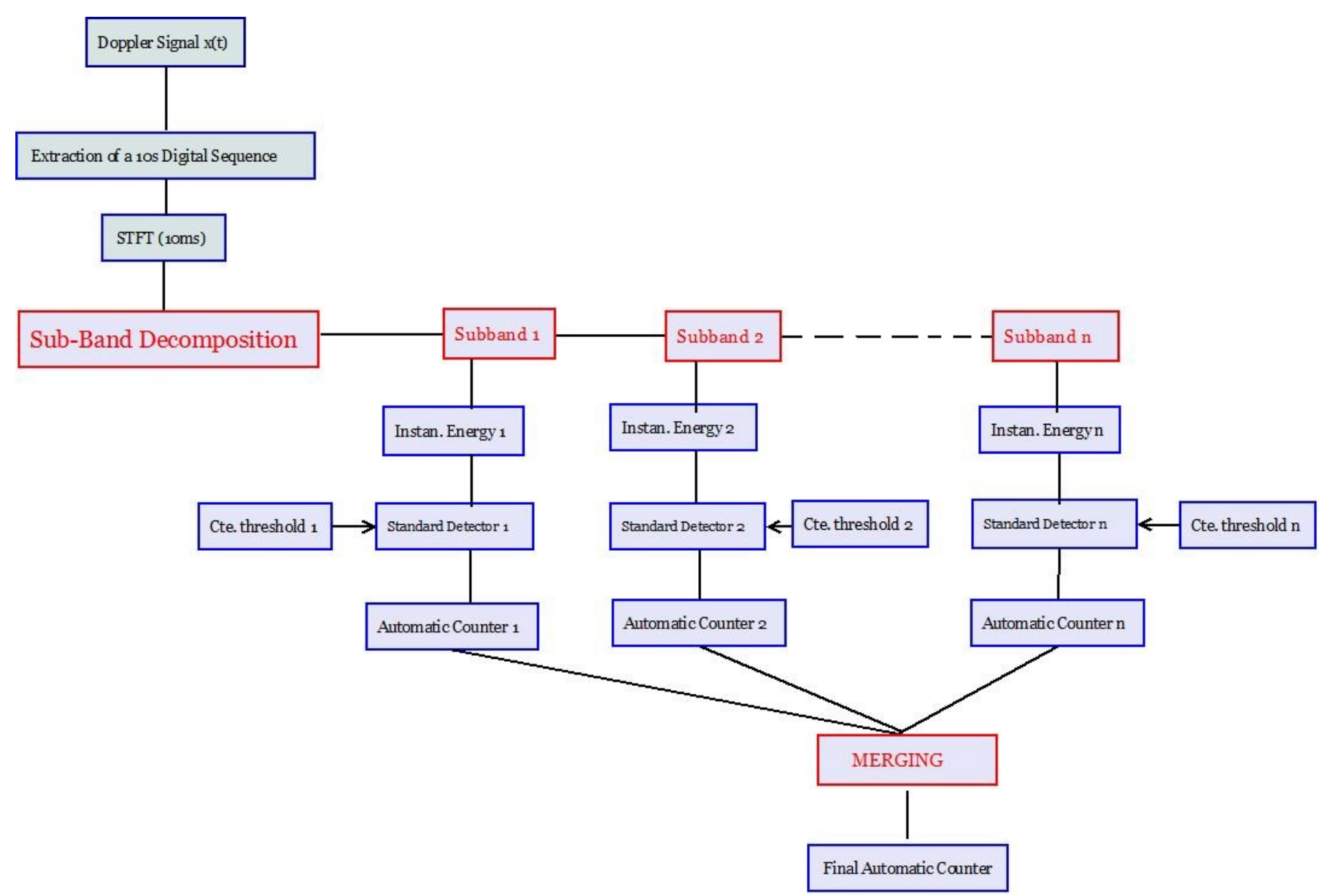

Figure 2: Sub-band Decomposition-based embolus detection system

Another comparison tool used to assess our results is the Embolus to Blood Ratio (EBR). EBR is the most widely used parameter in embolic signal detection. EBR indicates how strong an embolic signal is relative to the background Doppler signal. It can be calculated in the following manner:

$$
E B R=10 \log _{10}\left(\frac{P_{E+B}}{P_{B}}\right)
$$

$P_{E+B}$ is the backscattered power measured when an embolus and the blood are present in the Doppler sample volume. $P_{B}$ is the backscattered power measured from blood alone in the sample volume [8], [9].

\section{RESUlts}

As mentioned previously, our whole dataset is divided into 2 categories. We used the first to apply two different training phases and the second to apply the main phase called the testing phase. Below we demonstrate the work done in each phase and the associated results.

\section{A. Training Phase}

In the first training phase we use the first category of our dataset ( 8 signals) to determine the most suitable number of sub-bands to be used. The objective is to find the best trade-off between the total number of channels and the detection rate as well as the false alarm rate obtained. 2, 4 and 8 sub-bands are tested. The results are shown in Table I.

Through Table I, we deduce that the best detection procedure is obtained for number of sub-bands $N_{s b}=4$. Compared to 2 sub-bands, 4 sub-bands allows a better detection rate and a lower FAR. Compared to 8 sub-bands, 4 sub-bands allows a close detection rate with a much lower FAR.

In the second training phase we also use the first category of our dataset ( 8 signals) to determine the best empirical constant threshold values. The objective is to find the empirical threshold (in $\mathrm{dB}$ ), on one hand, for standard methods and on another hand for each sub-band of the 4 sub-band decomposition channels.

For standard methods, the best empirical threshold is found to be $5 \mathrm{~dB}$ above the background Doppler energy. For sub-band 
Table I: Results (Detection Rate and FAR) for 2, 4 and 8 sub-bands used

\begin{tabular}{|l|l|l|}
\hline $\begin{array}{l}\text { Number of Sub-band De- } \\
\text { composition }\end{array}$ & $\begin{array}{l}\text { Detection } \\
\text { Rate }(\%)\end{array}$ & FAR (\%) \\
\hline \hline 2 Sub-bands & 55.1 & 53.0 \\
\hline \hline 4 Sub-bands & 78.8 & 41.6 \\
\hline \hline 8 Sub-bands & 80.8 & 65.1 \\
\hline
\end{tabular}

Table II: Best empirical thresholds for standard detection and in each sub-band of the 4-sub-band decomposition

\begin{tabular}{|l|l|}
\hline $\begin{array}{l}\text { Number of Sub- } \\
\text { band Decomposi- } \\
\text { tion }\end{array}$ & $\begin{array}{l}\text { Best } \\
\text { Empirical } \\
\text { Threshold } \\
d B\end{array}$ \\
\hline $\begin{array}{l}\text { Standard } \\
\text { Detector }\end{array}$ & $5 d B$ \\
\hline $\begin{array}{l}\text { 4-Sub-band De- } \\
\text { tector Sub-band 1 }\end{array}$ & $4 d B$ \\
\hline $\begin{array}{l}\text { 4-Sub-band De- } \\
\text { tector Sub-band 2 }\end{array}$ & $4 d B$ \\
\hline $\begin{array}{l}\text { 4-Sub-band De- } \\
\text { tector Sub-band 3 }\end{array}$ & $5 d B$ \\
\hline $\begin{array}{l}\text { 4-Sub-band De- } \\
\text { tector Sub-band 4 }\end{array}$ & $3 d B$ \\
\hline
\end{tabular}

Table III: Comparison of the Detection rate, FAR, and EBR results for standard detectors and sub-band detectors

\begin{tabular}{|l|l|l|l|}
\hline Detector Type & $\begin{array}{l}\text { Detection } \\
\text { Rate }(\%)\end{array}$ & FAR $(\%)$ & EBR $(d B)$ \\
\hline $\begin{array}{l}\text { Standard } \\
\text { Detector }\end{array}$ & 66.0 & 43.7 & 24.4 \\
\hline $\begin{array}{l}\text { 4-Sub-band De- } \\
\text { tector }\end{array}$ & 78.9 & 36.5 & 39.6 \\
\hline
\end{tabular}

decomposition, the best empirical threshold in each sub-band is found to be $4 \mathrm{~dB}$ for sub-band 1 , $4 \mathrm{~dB}$ for sub-band 2 , $5 \mathrm{~dB}$ for sub-band 3 and $3 \mathrm{~dB}$ for sub-band 4 . The results are demonstrated in Table II.

\section{B. Testing Phase}

In this phase we use the second category of our dataset ( 9 signals) to determine the results of the two detectors used, the standard detector and the 4 sub-band detector. We compare the performance of each detector. The results are presented in Table III.

\section{Discussion AND CONCLUSION}

The results show that our sub-band detector allows increasing the detection rate by more than $12 \%$ and even reducing the FAR by more than $7 \%$. Consequently we affirm that a detector based on sub-band decomposition is more reliable and adapted than standard techniques. Moreover, the calculated EBRs for the two techniques show values of $24.4 d B$ for standard detectors compared to $39.6 d B$ for detectors based on sub-band decomposition. The EBR was increased by more than $15 d B$.

To conclude, in this study we have proposed an energy detector based on frequency sub-band decomposition through a bank of $\mathrm{n}$ ideal frequency filters, as a tool for robust micro-embolus detection. In a number of cases in which the micro-embolic signal can not be clearly distinguished from the background energy by whole band detection, it can be distinguished through sub-band filtering. The sub-band detector is composed of $n$ detectors associated to $n$ Doppler frequency subbands where the detection procedure is repeated in each and every sub-band simultaneously. The technique is compared to the standard techniques based on the detection over the whole Doppler frequency spectrum.

The results show that using sub-band decomposition allows better detection of micro-embolic events. The results confirm that sub-band decomposition reveals embolic signatures more apparently. It allows increasing the EBR and thus is in consistency with the widely known agreement that any processing method that improves the EBR would be able to ameliorate the performance of an automated embolus detection system [10]. In addition, the increase in the detection rate and the decrease in the number of false positive detections also emphasizes the efficiency of sub-band decomposition as a powerful method for micro-embolus detection. In the upcoming step, we will couple sub-band decomposition with time-varying thresholds, rather than using constant thresholds, in order to boost the overall performance of the detection system. 


\section{REFERENCES}

[1] W. H. O. WHO, World health statistics 2014. World Health Organization, 2014.

[2] Z. B. Jovanović, A. M. Pavlović, J. J. Zidverc-Trajković, M. D. Mijajlović, A. P. Radojičić, and N. M. Čovičković-Šternić, “Transcranial doppler test for evaluation of cerebral artery embolism: Microemboli detection," Srpski arhiv za celokupno lekarstvo, vol. 136, no. 5-6, pp. 302-306, 2008.

[3] L. L. Yeo and V. K. Sharma, "Role of transcranial doppler ultrasonography in cerebrovascular disease," Recent patents on CNS drug discovery, vol. 5, no. 1, pp. 1-13, 2010.

[4] N. Aydin, F. Marvasti, and H. S. Markus, "Embolic doppler ultrasound signal detection using discrete wavelet transform," IEEE Transactions on Information Technology in Biomedicine, vol. 8, no. 2, pp. 182-190, 2004.

[5] J.-M. Girault, "Cerebral microembolism synchronous detection with wavelet packets," Signal and Image Multiresolution Analysis, pp. $245-287,2013$.

[6] Y. Chen and Y. Wang, "Doppler embolic signal detection using the adaptive wavelet packet basis and neurofuzzy classification," Pattern recognition letters, vol. 29, no. 10, pp. 1589-1595, 2008.

[7] C. Lueang-on, C. Tantibundhit, and S. Muengtaweepongsa, "Abstract wmp60: Automatic embolic signal detection using adaptive wavelet packet transform and adaptive neuro-fuzzy inference system," Stroke, vol. 44, no. Suppl 1, pp. AWMP60-AWMP60, 2013.

[8] G. A. Darbellay, R. Duff, J.-M. Vesin, P.-A. Despland, D. W. Droste, C. Molina, J. Serena, R. Sztajzel, P. Ruchat, T. Karapanayiotides et al., "Solid or gaseous circulating brain emboli: are they separable by transcranial ultrasound?" Journal of cerebral blood flow \& metabolism, vol. 24 , no. 8, pp. 860-868, 2004.

[9] J.-M. Girault, D. Kouamé, A. Ouahabi, and F. Patat, "Micro-emboli detection: an ultrasound doppler signal processing viewpoint," IEEE Transactions on Biomedical Engineering, vol. 47, no. 11, pp. 1431-1439, 2000.

[10] D. H. Evans, "Ultrasonic detection of cerebral emboli," vol. 1, pp. 316-326, 2003. 